\title{
An Optimal Bound for Nonlinear Eigenvalues and Torsional Rigidity on Domains with Holes
}

\author{
Francesco Della Pietra and Gianpaolo Piscitelli
}

\begin{abstract}
In this paper we prove an optimal upper bound for the first eigenvalue of a Robin-Neumann boundary value problem for the $p$-Laplacian operator in domains with convex holes. An analogous estimate is obtained for the corresponding torsional rigidity problem.
\end{abstract}

Mathematics Subject Classification (2010). 35P15, 47J30, 35J92, 35J25.

Keywords. Nonlinear eigenvalue problems, torsional rigidity, mixed boundary conditions, optimal estimates.

\section{Introduction}

In this paper we prove an optimal bound for the first eigenvalue and the torsional rigidity of a Robin-Neumann boundary value problem for the $p$-Laplacian operator in domains with convex holes.

The problem of maximizing or minimizing elliptic functionals with different boundary conditions under various geometric constraints has been extensively studied. Based on the classical isoperimetric inequality, the first evidences are the celebrated Faber-Krahn inequality for the first Dirichlet eigenvalue of the Laplacian, or the Saint-Venant principle for the torsional rigidity with Dirichlet boundary conditions.

Let us consider $\Omega$ and $D$ two bounded open sets, with $D$ convex, $\Omega$ Lipschitz, connected, and $D \Subset \Omega$. We will denote by $\Sigma=\Omega \backslash \bar{D}$. Here we study the following problem:

$$
\lambda(\beta, \Sigma)=\min \left\{\frac{\int_{\Sigma}|\nabla \varphi|^{p} d x+\beta \int_{\partial D}|\varphi|^{p} d \sigma}{\int_{\Sigma}|\varphi|^{p} d x}, \varphi \in W^{1, p}(\Sigma), \varphi \not \equiv 0\right\},
$$


where $\beta$ is a positive constant and $1<p<+\infty$. A minimizer $u \in W^{1, p}(\Sigma)$ of $(1.1)$ satisfies

$$
\begin{cases}-\Delta_{p} u=\lambda(\beta, \Sigma)|u|^{p-2} u & \text { in } \Sigma, \\ |\nabla u|^{p-2} \frac{\partial u}{\partial \nu}=0 & \text { on } \partial \Omega, \\ |\nabla u|^{p-2} \frac{\partial u}{\partial \nu}+\beta|u|^{p-2} u=0 & \text { on } \partial D .\end{cases}
$$

In this paper we obtain an optimal upper bound on $\lambda(\beta, \Sigma)$, when $\beta>0$ is fixed and $\Sigma=\Omega \backslash \bar{D}$ varies among all domains such that the volume of $\Sigma$ and the $(n-1)$ quermassintegral of $D$ are given (see Section 2.3 for the precise definition). If $n=2$, the geometrical constraint on $D$ corresponds to fix its perimeter. In particular, we show that $\lambda(\beta, \Sigma)$ is maximized by the spherical shell. The first main result is the following.

Theorem 1.1. Let $\beta>0$, and $\Omega$ and $D$ be two bounded open sets, with $D$ convex, $\Omega$ Lipschitz, connected, and $D \Subset \Omega$. Let be $\Sigma=\Omega \backslash \bar{D}, A=A_{R_{1}, R_{2}}=B_{R_{2}} \backslash \bar{B}_{R_{1}}$, where $B_{R_{i}}$ is a ball centered at the origin with radius $R_{i}, i=1,2$. Suppose that $|A|=|\Sigma|$, and $W_{n-1}\left(B_{R_{1}}\right)=W_{n-1}(D)$. Then,

$$
\lambda(\beta, \Sigma) \leq \lambda(\beta, A) .
$$

In the case $p=n=2$, Theorem 1.1 recovers a result proved by Hersch in [18]. Our result generalizes it to the $p$-Laplacian operator and to any dimension, giving an answer to a question posed by Henrot in [17, Chapter 3, Open problem 5]. Actually, we are not able to prove or disprove that the first eigenvalue $\lambda(\beta, \Sigma)$ is maximized on the spherical shell when the perimeter of $D$, and not the $W_{n-1}$, is fixed. We stress that a related result has been recently proved for an optimal insulating problem (see $[14])$.

Optimal estimates for eigenvalues with different Robin or Robin-Neumann boundary conditions have been proved by several authors. For example, if $D=\emptyset$, and a Robin condition on $\partial \Omega$ is given, with $\beta>0$, a Faber-Krahn inequality has been proved in $[3,9]$ for $p=2$ and in $[5,8]$ for $p \in] 1,+\infty[$, stating that the first eigenvalue is minimum on the ball of the same volume of $\Omega$. Otherwise, if $\beta$ is negative the problem is still open; in this direction, in [15] the authors showed that, among planar domains of the same measure, the disk is a maximizer only for small value of the parameter. On the other hand, if the perimeter rather than the volume is fixed, the ball maximizes the first eigenvalue among all open, bounded, convex, smooth enough sets (see $[2,6]$ ).

In this framework, the case of Robin eigenvalue problems when $\beta \geq 0$ is not a constant has been considered in [16], and, for example, in $[4,13,11]$ where optimization with respect to $\beta$ is considered.

Coming back to the problem with mixed boundary conditions, we recall that the case of a Neumann condition on $\partial D$ and Robin condition on $\partial \Omega$ has been considered in $[22,20]$. 
Another problem we deal with is the $p$-torsional rigidity with the same boundary conditions, namely

$$
T(\beta, \Sigma)=\max \left\{\frac{\left(\int_{\Sigma}|\varphi| d x\right)^{p}}{\int_{\Sigma}|\nabla \varphi|^{p} d x+\beta \int_{\partial D}|\varphi|^{p} d \sigma}, \varphi \in W^{1, p}(\Sigma), \varphi \not \equiv 0\right\},
$$

with $\beta>0$. The unique function $u_{\Sigma}>0$ such that

$$
\begin{cases}-\Delta_{p} u_{\Sigma}=1 & \text { in } \Sigma \\ \left|\nabla u_{\Sigma}\right|^{p-2} \frac{\partial u_{\Sigma}}{\partial \nu}=0 & \text { on } \partial \Omega \\ \left|\nabla u_{\Sigma}\right|^{p-2} \frac{\partial u_{\Sigma}}{\partial \nu}+\beta\left|u_{\Sigma}\right|^{p-2} u_{\Sigma}=0 & \text { on } \partial D\end{cases}
$$

is a maximizer of (1.3), and

$$
T(\beta, \Sigma)=\left(\int_{\Sigma} u_{\Sigma} d x\right)^{p-1} .
$$

Moreover, any maximizer of (1.3) is proportional to $u_{\Sigma}$.

We prove that the spherical shell minimizes $T(\beta, \Sigma)$ among all domains such that the volume of $\Sigma$ and the $(n-1)$-quermassintegral of $D$ are given.

Theorem 1.2. Let $\beta>0$, and $\Omega$ and $D$ be two bounded open sets, with $D$ convex, $\Omega$ Lipschitz, connected, and $D \Subset \Omega$. Let be $\Sigma=\Omega \backslash \bar{D}, A=A_{R_{1}, R_{2}}=B_{R_{2}} \backslash \bar{B}_{R_{1}}$, where $B_{R_{i}}$ is a ball centered at the origin with radius $R_{i}, i=1,2$. Suppose that $|A|=|\Sigma|$, and $W_{n-1}\left(B_{R_{1}}\right)=W_{n-1}(D)$. Then,

$$
T(\beta, \Sigma) \geq T(\beta, A) .
$$

Regarding optimal estimates related to the torsional rigidity when $D=\emptyset$ and a Robin boundary condition is given on $\partial \Omega$, a Saint-Venant inequality can be proved: we refer the reader to $[1,7]$.

Finally, we recall that in this context, estimates have been obtained also for a more general class of problems, involving the so called Finsler operator. We refer the reader, for example, to $[10,12,21]$.

The structure of the paper is the following. In the second section, we prove some properties of the first eigenvalue $\lambda(\beta, \Sigma)$ and of the torsional rigidity $T(\beta, \Sigma)$, as well as we recall some basic tool of convex analysis. In the third section, we prove the main results. We conclude the paper with a section containing the possible future directions.

\section{Notation and preliminaries}

\subsection{Eigenvalue problem}

The following result can be proved by a standard argument of Calculus of Variations. 
Proposition 2.1. Let $\beta>0, D, \Omega$ Lipschitz domains with $D \Subset \Omega$, and $\Sigma=\Omega \backslash \bar{D}$.

- There exists a positive minimizer $u \in W^{1, p}(\Sigma)$ of (1.1). Moreover, $u$ is a solution of (1.2).

- The eigenvalue $\lambda(\beta, \Sigma)$ is simple, that is all the associated eigenfunctions are scalar multiple of each other.

A trivial upper bound for $\lambda(\beta, \Sigma)$ is given, by choosing constant test functions, by

$$
\lambda(\beta, \Sigma) \leq \beta \frac{P(D)}{|\Sigma|} .
$$

Another simple upper bound for $\lambda(\beta, \Sigma)$ is given by

$$
\lambda(\beta, \Sigma) \leq \Lambda(\Sigma)
$$

where

$$
\Lambda(\Sigma)=\min \left\{\frac{\int_{\Sigma}|\nabla \varphi|^{p} d x}{\int_{\Sigma}|\varphi|^{p} d x}, \varphi \in W^{1, p}(\Sigma),\left.\varphi\right|_{\partial D}=0\right\},
$$

that is the first eigenvalue of the corresponding Dirichlet-Neumann problem.

In the radial case, we have that the eigenfunctions are radially symmetric and strictly increasing in the radial variable, as stated in the following

Proposition 2.2. Let be $\beta>0, R_{2}>R_{1}>0$ and let $v$ be a positive minimizer of problem (1.1) on the spherical shell $\Sigma=A_{R_{1}, R_{2}}$. Then $v$ is radially symmetric, in the sense that $v(x)=\psi(|x|)$. Moreover, $\psi^{\prime}(r)>0$.

Proof. The radial symmetry of the minimizers follows from the rotational invariance of problem (1.1) and the simplicity of $\lambda\left(\beta, A_{R_{1}, R_{2}}\right)$.

Now, let $\psi$ be a positive radial eigenfunction. We observe that $\psi$ solves

$$
\left\{\begin{array}{l}
-\frac{1}{r^{n-1}}\left(\left|\psi^{\prime}(r)\right|^{p-2} \psi^{\prime}(r) r^{n-1}\right)^{\prime}=\lambda\left(\beta, A_{R_{1}, R_{2}}\right) \psi^{p-1}(r) \text { if } r \in\left(R_{1}, R_{2}\right), \\
\psi^{\prime}\left(R_{2}\right)=0, \\
\left|\psi^{\prime}\left(R_{1}\right)\right|^{p-2} \psi^{\prime}\left(R_{1}\right)+\beta \psi^{p-1}\left(R_{1}\right)=0 .
\end{array}\right.
$$

For every $r \in\left(R_{1}, R_{2}\right)$ it holds that

$$
-\frac{1}{r^{n-1}}\left(\left|\psi^{\prime}(r)\right|^{p-2} \psi^{\prime}(r) r^{n-1}\right)^{\prime}=\lambda\left(\beta, A_{R_{1}, R_{2}}\right) \psi^{p-1}(r)>0,
$$

and, as a consequence,

$$
\left(\left|\psi^{\prime}(r)\right|^{p-2} \psi^{\prime}(r) r^{n-1}\right)^{\prime}<0
$$

Taking into account the boundary conditions $\psi^{\prime}\left(R_{2}\right)=0$, it follows by integrating that $\psi^{\prime}(r)>0$, and this concludes the proof.

The following proposition is a complementary result which states some properties of the first eigenvalue $\lambda(\beta, \Sigma)$, related to its dependence on the parameter $\beta$. 
Proposition 2.3. Let $\beta>0, D, \Omega$ Lipschitz domains with $D \Subset \Omega$, and $\Sigma=\Omega \backslash \bar{D}$. The following properties hold.

1. The first eigenvalue $\lambda(\beta, \Sigma)$ is differentiable and nondecreasing in $\beta>0$. Moreover,

$$
\frac{d}{d \beta} \lambda(\beta, \Sigma)=\frac{\int_{\partial D}|u|^{p} d \sigma}{\int_{\Sigma}|u|^{p} d x}
$$

where $u$ is a minimizer of (1.1).

2. It holds that

$$
\lim _{\beta \rightarrow+\infty} \lambda(\beta, \Sigma)=\Lambda(\Sigma) .
$$

3. The first eigenvalue $\lambda(\beta, \Sigma)$ is concave with respect to $\beta$.

Proof. Let us denote

$$
Q(\beta, \varphi)=\frac{\int_{\Sigma}|\nabla \varphi|^{p} d x+\beta \int_{\partial D}|\varphi|^{p} d \sigma}{\int_{\Sigma}|\varphi|^{p} d x}, \quad \varphi \in W^{1, p}(\Sigma) .
$$

Let $u_{\beta}, u_{\beta+h}$ be two positive minimizers of (1.1) associated to $\beta$ and $\beta+h$, respectively. Moreover, suppose $\int_{\Sigma} u_{\beta}^{p} d x=\int_{\Sigma} u_{\beta+h}^{p} d x=1$. Then,

$$
\lambda(\beta+h, \Sigma)-\lambda(\beta, \Sigma) \geq \lambda(\beta+h, \Sigma)-Q\left(\beta, u_{\beta+h}\right)=h \int_{\partial D} u_{\beta+h}^{p} d \sigma .
$$

On the other hand,

$$
\lambda(\beta+h, \Sigma)-\lambda(\beta, \Sigma) \leq Q\left(\beta+h, u_{\beta}\right)-\lambda(\beta, \Sigma)=h \int_{\partial D} u_{\beta}^{p} d \sigma .
$$

Finally,

$$
\int_{\partial D} u_{\beta+h}^{p} d \sigma \leq \frac{\lambda(\beta+h, \Sigma)-\lambda(\beta, \Sigma)}{h} \leq \int_{\partial D} u_{\beta}^{p} d \sigma .
$$

Then, being $\left\{u_{\beta+h}\right\}_{h}$ bounded in $W^{1, p}(\Omega)$, there exists a subsequence, still denoted by $u_{\beta+h}$ such that $u_{\beta+h} \rightarrow u_{\beta}$ strongly in $L^{p}$ and almost everywhere and $\nabla u_{\beta+h} \rightarrow$ $\nabla u_{\beta}$ weakly in $L^{p}(\Omega)$. As a consequence, by the compactness of the trace operator (see for example [19, Cor. 18.4]), $u_{\beta+h}$ converges strongly to $u_{\beta}$ in $L^{p}(\partial D)$.

The monotonicity of $\lambda(\beta, \Sigma)$ with respect to $\beta$ is obvious.

Now we show the continuity of $\lambda$ with respect to $\beta$. Define

$$
Q(\beta, \varphi)=\frac{\int_{\Sigma}|\nabla \varphi|^{p} d x+\beta \int_{\partial D}|\varphi|^{p} d \sigma}{\int_{\Sigma}|\varphi|^{p} d x}, \quad \varphi \in W^{1, p}(\Sigma) .
$$

Let $\varphi_{k}$ be a minimizing sequence for $\lambda(\beta, \Sigma)$, normalized with $\left\|\varphi_{k}\right\|_{L^{p}(\Sigma)}=1$. Then $\int_{\partial D} \varphi_{k} d \sigma \leq C=C(\Sigma, \beta)$. Hence

$$
\lambda(\beta, \Sigma) \leq \lambda(\beta+\varepsilon, \Sigma) \leq Q\left(\beta+\varepsilon, \varphi_{k}\right) \leq Q\left(\beta, \varphi_{k}\right)+\varepsilon C .
$$


Passing to the limit as $k \rightarrow+\infty$, we get

$$
0 \leq \lambda(\beta+\varepsilon, \Sigma)-\lambda(\beta, \Sigma) \leq \varepsilon C .
$$

Let $u_{\beta}$ be a minimizer for $\lambda(\beta, \Sigma)$, with $\int_{\Sigma}\left|u_{\beta}\right|^{p} d x=1$. Then being

$$
\lambda(\beta, \Sigma)=\int_{\Sigma}\left|\nabla u_{\beta}\right|^{p} d x+\beta \int_{\partial D}\left|u_{\beta}\right|^{p} d \sigma \leq \lambda(\Sigma)
$$

if $\beta_{j} \rightarrow+\infty$ as $j \rightarrow+\infty$, there exists $u_{\infty} \in W^{1, p}(\Sigma)$ such that, up to a subsequence,

$$
u_{\beta_{j}} \rightarrow u_{\infty} \text { in } W^{1, p}(\Sigma), u_{\beta_{j}} \rightarrow u_{\infty} \equiv 0 \text { in } L^{p}(\partial D), u_{\beta_{j}} \rightarrow u_{\infty} \text { in } L^{p}(\Sigma) .
$$

Hence by lower semicontinuity,

$$
\lambda(\Sigma) \leq \int_{\Sigma}\left|\nabla u_{\infty}\right|^{p} d x \leq \liminf _{j} \lambda\left(\beta_{j}, \Sigma\right) \leq \lambda(\Sigma),
$$

that gives (2.1).

We finally prove that $\lambda(\beta, \Sigma)$ is concave in $\beta$. Indeed, for fixed $\beta_{0} \in \mathbb{R}$, we have to show that

$$
\lambda(\beta, \Sigma) \leq \lambda\left(\beta_{0}, \Sigma\right)+\frac{d}{d \beta} \lambda\left(\beta_{0}, \Omega\right)\left(\beta-\beta_{0}\right),
$$

for every $\beta>0$. Let $u_{0}$ the eigenfunction associated to $\lambda\left(\beta_{0}, \Sigma\right)$ and normalized such that $\int_{\Sigma} u_{0}^{p} d x=1$. Hence, we have

$$
\lambda(\beta, \Sigma) \leq \int_{\Sigma}\left|D u_{0}\right|^{p} d x+\beta \int_{\partial D}\left|u_{0}\right|^{p} d \mathcal{H}^{n-1} .
$$

Now, summing and subtracting to the right hand side of (2.3), $\beta_{0} \int_{\partial D}\left|u_{0}\right|^{p} d \mathcal{H}^{n-1}$, taking into account that we have just showed that

$$
\frac{d}{d \beta} \lambda\left(\beta_{0}, \Omega\right)=\int_{\partial D}\left|u_{0}\right|^{p} d \mathcal{H}^{n-1},
$$

we obtain (2.2).

\subsection{The $p$-torsional rigidity}

Similarly to the case of the first eigenvalue, the following results hold.

Proposition 2.4. Let $\beta>0$. There exists a unique positive maximizer $u_{\Sigma} \in W^{1, p}(\Sigma)$ of (1.3) which solves (1.4), and any other maximizer of (1.4) is a scalar multiple of $u_{\Sigma}$.

Proposition 2.5. Let be $\beta>0, R_{2}>R_{1}>0$ and let $w$ be a positive maximizer of problem (1.3) on the spherical shell $\Sigma=A_{R_{1}, R_{2}}$. Then $w$ is radially symmetric, $w(x)=\Psi(|x|)$, with $\Psi^{\prime}(r)>0$. 


\subsection{Quermassintegrals and the Aleksandrov-Fenchel inequalities}

Here we list some basic properties of convex analysis which will be useful in the following. For an extended discussion on the subject we refer the reader to [23].

Let $K$ be a bounded convex open set, and $B_{1}=\{x:|x|<1\}$. The outer parallel body of $K$ at distance $\rho>0$ is the Minkowski sum

$$
K+\rho B_{1}=\left\{x+\rho y \in \mathbb{R}^{n} \mid x \in K, y \in B_{1}\right\} .
$$

The Steiner formulas assert that

$$
\left|K+\rho B_{1}\right|=\sum_{i=0}^{n}\left(\begin{array}{l}
n \\
i
\end{array}\right) W_{i}(K) \rho^{i}
$$

and

$$
P\left(K+\rho B_{1}\right)=n \sum_{i=0}^{n-1}\left(\begin{array}{c}
n-1 \\
i
\end{array}\right) W_{i+1}(K) \rho^{i} .
$$

The coefficients $W_{i}(K)$ are known as the quermassintegrals of $K$. In particular, it holds that

$$
W_{0}(K)=|K|, \quad n W_{1}(K)=P(K), \quad W_{n}(K)=\omega_{n} .
$$

The Aleksandrov-Fenchel inequalities state that

$$
\left(\frac{W_{j}(K)}{\omega_{n}}\right)^{\frac{1}{n-j}} \geq\left(\frac{W_{i}(K)}{\omega_{n}}\right)^{\frac{1}{n-i}}, \quad 0 \leq i<j \leq n-1,
$$

where the inequality is replaced by an equality if and only if $K$ is a ball.

In what follows, we use the Aleksandrov-Fenchel inequalities for particular values of $i$ and $j$. When $i=0$ and $j=1$, we have the classical isoperimetric inequality:

$$
P(K) \geq n \omega_{n}^{\frac{1}{n}}|K|^{1-\frac{1}{n}}
$$

Let us denote by $K^{*}$ a ball such that $W_{n-1}(K)=W_{n-1}\left(K^{*}\right)$. Then by AleksandrovFenchel inequalities (2.4), for $0 \leq i<n-1$

$$
\left(\frac{W_{i}(K)}{\omega_{n}}\right)^{\frac{1}{n-i}} \leq \frac{W_{n-1}(K)}{\omega_{n}}=\left(\frac{W_{i}\left(K^{*}\right)}{\omega_{n}}\right)^{\frac{1}{n-i}}
$$

hence

$$
W_{i}(K) \leq W_{i}\left(K^{*}\right), \quad 0 \leq i<n-1
$$

\section{Proof of main results}

In this section we prove the main results (Theorem 1.1 and Theorem 1.2).

Proof of Theorem 1.1. Let $v(x)=\psi(|x|)$ be a positive radial solution of problem (1.1) on $A$, and denote by $v_{m}=\psi\left(R_{1}\right)$ and $v_{M}=\psi\left(R_{2}\right)$ be the minimum and maximum of $v$, respectively.

For $x \in \Sigma$, let us denote by $d(x)$ the distance of $x$ from $D$,

$$
d(x)=\inf \{|x-y|, y \in D\},
$$


and consider as test function

$$
w(x):= \begin{cases}G(d(x)) & \text { if } d(x)<R_{2}-R_{1}, \\ v_{M} & \text { if } d(x) \geq R_{2}-R_{1}\end{cases}
$$

where $G$ is defined as

$$
G^{-1}(t)=\int_{v_{m}}^{t} \frac{1}{g(\tau)} d \tau, \quad v_{m}<t<v_{M},
$$

with $g(t)=|D v|_{v=t}$. We observe that $v(x)=G\left(|x|-R_{1}\right)$ and $w$ satisfies the following properties:

$$
\begin{gathered}
w \in W^{1, p}(\Sigma) \cap C(\bar{\Sigma}), \\
|D w|_{w=t}=|D v|_{v=t}, \\
w_{m}:=\min _{\bar{\Sigma}} w=v_{m}=G(0), \\
w_{M}:=\max _{\bar{\Sigma}} w=v_{M} .
\end{gathered}
$$

Since $R_{2}-R_{1}=G^{-1}\left(v_{M}\right)$, to prove that the maximum value of $w$ is $v_{M}$, we need to verify that $G^{-1}\left(v_{M}\right)=\int_{v_{m}}^{v_{M}} \frac{1}{g(\tau)} d \tau$. Indeed since $v(x)=\psi(|x|)$, then $\int_{v_{m}}^{v_{M}} \frac{1}{\psi^{\prime}\left(v^{-1}(s)\right)} d s=$ $\psi^{-1}\left(v_{M}\right)-\psi^{-1}\left(v_{m}\right)=R_{2}-R_{1}$.

Let us denote

$$
E_{t}=\bar{D} \cup\{x \in \Sigma: w<t\}, \quad F_{t}=\bar{B}_{R_{1}} \cup\{x \in A: v<t\} .
$$

Observe that

$$
E_{t} \subseteq\left\{x \in \mathbb{R}^{n}: d(x)<G^{-1}(t)\right\}:=\tilde{E}_{t}, \quad F_{t}=\left\{x \in \mathbb{R}^{n}:|x|<R_{1}+G^{-1}(t)\right\} .
$$

By the Steiner formula and the Aleksandrov-Fenchel inequalities, we get, as $\rho=$ $G^{-1}(t)$, that

$$
\begin{aligned}
\mathcal{H}^{n-1}\left(\partial E_{t} \cap \Sigma\right) & \leq P\left(\tilde{E}_{t}\right)=P\left(D+\rho B_{1}\right)=n \sum_{k=0}^{n-1}\left(\begin{array}{c}
n-1 \\
k
\end{array}\right) W_{k+1}(D) \rho^{k} \\
& \leq n \sum_{k=0}^{n-1}\left(\begin{array}{c}
n-1 \\
k
\end{array}\right) W_{k+1}\left(B_{R_{1}}\right) \rho^{k}=P\left(B_{R_{1}}+\rho B_{1}\right)=P\left(F_{t}\right) .
\end{aligned}
$$

Using now the coarea formula and (3.1):

$$
\begin{aligned}
\int_{\Omega}|\nabla w|^{p} d x & =\int_{w_{m}}^{w_{M}} g(t)^{p-1} \mathcal{H}^{n-1}\left(\partial E_{t} \cap \Sigma\right) d t \\
& \leq \int_{w_{m}}^{w_{M}} g(t)^{p-1} P\left(\tilde{E}_{t}\right) d t \leq \int_{v_{m}}^{v_{M}} g(t)^{p-1} P\left(F_{t}\right) d t=\int_{A}|\nabla v|^{p} d x .
\end{aligned}
$$

Since, by construction, $w(x)=w_{m}=v_{m}$ on $\partial D$, then

$$
\int_{\partial D} w^{p} d \mathcal{H}^{n-1}=w_{m}^{p} P(D) \leq v_{m}^{p} P\left(B_{R_{1}}\right)=\int_{\partial B_{R_{1}}} v^{p} d \mathcal{H}^{n-1} .
$$


Now, we define $\mu(t)=\left|E_{t} \cap \Sigma\right|$ and $\eta(t)=\left|F_{t}\right|$ and using again coarea formula, we obtain, for $v_{m} \leq t<v_{M}$,

$$
\begin{aligned}
\mu^{\prime}(t)=\int_{\{w=t\} \cap \Sigma} \frac{1}{|\nabla w(x)|} d \mathcal{H}^{n-1} & =\frac{\mathcal{H}^{n-1}\left(\partial E_{t} \cap \Sigma\right)}{g(t)} \leq \frac{P\left(\tilde{E}_{t}\right)}{g(t)} \\
& \leq \frac{P\left(F_{t}\right)}{g(t)}=\int_{\{v=t\}} \frac{1}{|\nabla v(x)|} d \mathcal{H}^{n-1}=\eta^{\prime}(t)
\end{aligned}
$$

This inequality is trivially true also if $0<t<v_{m}$. Since $\mu(0)=\eta(0)=0$, by integrating from 0 to $t<v_{M}$, we have:

$$
\mu(t) \leq \eta(t)
$$

On the other hand, we have

$$
\begin{aligned}
\int_{\Sigma} w^{p} d x & =\int_{0}^{v_{M}} p t^{p-1}(|\Sigma|-\mu(t)) d t \\
& \geq \int_{0}^{v_{M}} p t^{p-1}(|A|-\eta(t)) d t=\int_{A} v^{p} d x .
\end{aligned}
$$

Using (3.2), (3.3) and (3.4), we achieve

$$
\begin{aligned}
\lambda(\beta, \Sigma) \leq & \frac{\int_{\Sigma}|\nabla w|^{p} d x+\beta \int_{\partial D} w^{p} d \mathcal{H}^{n-1}}{\int_{\Sigma} w^{p} d x} \\
& \leq \frac{\int_{A}|\nabla v|^{p} d x+\beta \int_{\partial B_{R_{1}}} v^{p} d \mathcal{H}^{n-1}}{\int_{A} v^{p} d x}=\lambda(\beta, A),
\end{aligned}
$$

and this concludes the proof.

Proof of Theorem 1.2. As regards the case of the torsional rigidity, the proof follows line by line the proof of Theorem 1.1 making use of the stress function $v_{A}$ instead of the first eigenfunction on the spherical shell.

Remark 3.1. We stress that with the same argument it is possible to prove that if

$$
\lambda_{q}(\beta, \Sigma)=\min \left\{\frac{\int_{\Sigma}|\nabla \varphi|^{p} d x+\beta \int_{\partial D}|\varphi|^{p} d \sigma}{\left(\int_{\Sigma}|\varphi|^{q} d x\right)^{\frac{p}{q}}}, \varphi \in W^{1, p}(\Sigma), \varphi \not \equiv 0\right\},
$$

with $1 \leq q \leq p$ and $1<p<+\infty$, it holds that

$$
\lambda_{q}(\beta, \Sigma) \leq \lambda_{q}(\beta, A)
$$

where $A=B_{R_{2}} \backslash \bar{B}_{R_{1}}$ is the spherical shell with $|A|=|\Sigma|$ and $W_{n-1}\left(B_{R_{1}}\right)=$ $W_{n-1}(D)$. 


\section{Conclusions}

In conclusion, we have considered the first eigenvalue and the torsional rigidity of the $p$-Laplacian operator, defined in a bounded Lipschitz domain of $\mathbb{R}^{n}$ with a convex hole, where a Robin boundary condition on the hole and a Neumann condition on the other parts of the boundary is given. We have proved that the first eigenvalue of the spherical shell is maximal among the domains with fixed volume and fixed $(n-1)$-quermassintegral of the hole, while the torsional rigidity is minimal. It is still an open problem to understand what happens if instead of $W_{n-1}$ the perimeter or the other $k$-th quermassintegrals of the hole, are fixed. Further developments of the present work could go in this direction.

Another possible direction of investigation is to understand if this kind of results may be generalized to different kind of operators, as the Finsler Laplacian or, more generally, the Finsler $p$-Laplacian (see for example $[10,12]$ and the references therein contained). In this case, is reasonable that quermassintegrals with respect to the Finsler metric will be involved, and the candidate as optimal set will be the spherical shell with respect to the polar norm of the considered Finsler metric.

Acknowledgements. This work has been partially supported by a MIUR-PRIN 2017 grant "Qualitative and quantitative aspects of nonlinear PDE's" and by GNAMPA of INdAM. The second author (G.P.) was also supported by Progetto di eccellenza "Sistemi distribuiti intelligenti" of Dipartimento di Ingegneria Elettrica e dell'Informazione "M. Scarano".

Funding. Open access funding provided by Università degli Studi di Napoli Federico II within the CRUI-CARE Agreement.

Open Access. This article is licensed under a Creative Commons Attribution 4.0 International License, which permits use, sharing, adaptation, distribution and reproduction in any medium or format, as long as you give appropriate credit to the original author(s) and the source, provide a link to the Creative Commons licence, and indicate if changes were made. The images or other third party material in this article are included in the article's Creative Commons licence, unless indicated otherwise in a credit line to the material. If material is not included in the article's Creative Commons licence and your intended use is not permitted by statutory regulation or exceeds the permitted use, you will need to obtain permission directly from the copyright holder. To view a copy of this licence, visit http://creativecommons.org/licenses/by/4.0/.

Publisher's Note. Springer Nature remains neutral with regard to jurisdictional claims in published maps and institutional affiliations.

\section{References}

[1] A. Alvino, C. Nitsch, C. Trombetti, A Talenti comparison result for solutions to elliptic problems with Robin boundary conditions, ArXiv (2019). 
[2] P.R. Antunes, P. Freitas, D. Krejčiřík, Bounds and extremal domains for Robin eigenvalues with negative boundary parameter, Adv. Calc. Var. 10 (2017), 357-379.

[3] M.H. Bossel, Membranes élastiquement liées: extension du theoreme de Rayleigh-FaberKrahn t de l'inegalité de Cheeger, C.R. Acad. Sci. Paris Sér. I Math. 302 (1986), 47-50.

[4] D. Bucur, G. Buttazzo, C. Nitsch, Symmetry breaking for a problem in optimal insulation, J. Math. Pures Appl. 107 (2017), 451-463.

[5] D. Bucur, D. Daners, An alternative approach to the Faber-Krahn inequality for Robin problems, Calc. Var. Partial Differential Equations 37 (2010), 37-75.

[6] D. Bucur, V. Ferone, C. Nitsch, C. Trombetti, A sharp estimate for the first RobinLaplacian eigenvalue with negative boundary parameter, Atti Accad. Naz. Lincei Rend. Lincei Mat. Appl. 30 (2019), 665-676.

[7] D. Bucur, A. Giacomini, The Saint-Venant inequality for the Laplace operator with Robin boundary conditions, Milan J. Math. 83 (2015), 327-343.

[8] Q. Dai, Y. Fu, Faber-Krahn inequality for Robin problems involving p-Laplacian, Acta Math. Appl. Sin. Engl. Ser. 27 (2011), 13-28.

[9] D. Daners, A Faber-Krahn inequality for Robin problems in any space dimension, Math. Ann. 335 (2006), 767-785.

[10] F. Della Pietra, N. Gavitone, Faber-Krahn inequality for anisotropic eigenvalue problems with Robin boundary conditions, Potential Anal. 41 (2014), 1147-1166.

[11] F. Della Pietra, N. Gavitone, H. Kovařík, Optimizing the first eigenvalue of some quasilinear operators with respect to the boundary conditions, ESAIM Control Optim. Calc. Var. 23 (2017), 1381-1395.

[12] F. Della Pietra, N. Gavitone, G. Piscitelli, A sharp weighted anisotropic Poincaré inequality for convex domains, C.R. Acad. Sci. Paris 355 (2017), 748-752.

[13] F. Della Pietra, C. Nitsch, R. Scala, C. Trombetti, An optimization problem in thermal insulation with Robin boundary conditions, preprint, ArXiv (2020), https://arxiv.org/abs/2008.02193.

[14] F. Della Pietra, C. Nitsch, C. Trombetti, An optimal insulation problem, Math. Ann. (2020), https://doi.org/10.1007/s00208-020-02058-6

[15] P. Freitas, D. Krejčiř́k, The first Robin eigenvalue with negative boundary parameter, Adv. Math. 280 (2015), 322-339.

[16] N. Gavitone, L. Trani, On the First Robin Eigenvalue of a Class of Anisotropic Operators, Milan J. Math. 86 (2018), 201-223.

[17] A. Henrot, Extremum problems for eigenvalues of elliptic operators, Frontiers in Mathematics, Birkhäuser, Basel, 2006.

[18] J. Hersch, Contribution to the method of interior parallels applied to vibrating membranes, in: Studies in Mathematical Analysis and Related Topics, Stanford University Press, 1962, pp. 132-139.

[19] G. Leoni, A first course in Sobolev spaces, 2nd ed., Graduate Studies in Mathematics, vol. 181, American Mathematical Society, Providence, RI, 2017.

[20] G. Paoli, G. Piscitelli, L. Trani, textitSharp estimates for the first $p$-Laplacian eigenvalue and for the $p$-torsional rigidity on convex sets with holes. ArXiv (2019).

[21] G. Paoli, L. Trani, Two estimates for the first Robin eigenvalue of the Finsler Laplacian with negative boundary parameter, J. Optim. Theory Appl. 181 (2019), 743-757. 
[22] L.E. Payne, H.F. Weinberger, Some isoperimetric inequalities for membrane frequencies and torsional rigidity, J. Math. Anal. Appl. 2 (1961), 210-216.

[23] R. Schneider, Convex bodies: the Brunn-Minkowski theory. Second expanded edition, Encyclopedia of Mathematics and its Applications, vol. 151, Cambridge University Press, Cambridge, 2014.

Francesco Della Pietra

Dipartimento di Matematica e Applicazioni "R. Caccioppoli"

Università degli studi di Napoli Federico II

Via Cintia, Monte S. Angelo, 80126 Napoli, Italia.

e-mail: f.dellapietra@unina.it

Gianpaolo Piscitelli

Dipartimento di Ingegneria Elettrica e dell'Informazione "M. Scarano"

Università degli Studi di Cassino e del Lazio Meridionale

Via G. Di Biasio n. 43, 03043 Cassino (FR), Italy.

e-mail: gianpaolo.piscitelli@unicas.it

Received: June 22, 2020.

Accepted: July 31, 2020. 